\title{
«Expressions maghrébines», vol. 14, n. 1
}

\section{Carminella Biondi}

\section{Q OpenEdition}

\section{Journals}

\section{Edizione digitale}

URL: http://journals.openedition.org/studifrancesi/5455

DOI: 10.4000/studifrancesi.5455

ISSN: 2421-5856

\section{Editore}

Rosenberg \& Sellier

\section{Edizione cartacea}

Data di pubblicazione: 1 dicembre 2016

Paginazione: $572-574$

ISSN: 0039-2944

\section{Notizia bibliografica digitale}

Carminella Biondi, « «Expressions maghrébines», vol. 14, n. 1 », Studi Francesi [Online], 180 (LX | III) | 2016, online dal 01 janvier 2017, consultato il 18 septembre 2020. URL : http://

journals.openedition.org/studifrancesi/5455; DOI : https://doi.org/10.4000/studifrancesi.5455

Questo documento è stato generato automaticamente il 18 settembre 2020.

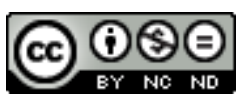

Studi Francesi è distribuita con Licenza Creative Commons Attribuzione - Non commerciale - Non opere derivate 4.0 Internazionale. 


\section{«Expressions maghrébines», vol. 14, n. 1}

\section{Carminella Biondi}

\section{NOTIZIA}

«Expressions maghrébines», vol. 14, n. 1, été 2015: «Écrivaines d'Algérie: fictions de l'origine, origines de la fiction». Dossier coordonné par Joana MAsò et Gemma VENTURA MUSTIENES, $198 \mathrm{pp}$.

1 Le motivazioni, le modalità di ricerca e le finalità del dossier si desumono subito, come capita quasi sempre, in particolare nelle raccolte miscellanee, dall'incipit dell'introduzione: «L'ensemble de réflexions qui configurent ce dossier prennent au sérieux l'espace littéraire comme producteur d'imaginaire commun par les biais de différentes matrices théoriques relevant de la critique littéraire, la philosophie, l'esthétique, les études postcoloniales ou de genre. Une matrice critique ici consacrée à étudier la généalogie et l'héritage historico-littéraires mais aussi esthétiques dans l'œuvre de quelques écrivaines d'Algérie: Fadila al-Faruq, Latifa Ben Mansour, Maïssa Bey, Nina Bouraoui, Hélène Cixous, Assia Djébar, Malika Mokeddem, Ahlam Mosteghanemi, Leilla Sebbar et la philosophe Catherine Malabou» (p. 1). Una «matrice critique» molto ampia (anche se Derrida costituisce la tela di fondo di alcuni di questi saggi), dunque un progetto che potrebbe incorrere in rischi di dispersione se l'unità non fosse assicurata dall'oggetto di ricerca: la "genealogia" letteraria di un gruppo di scrittrici algerine, quasi tutte di lingua francese o tradotte in francese (Fadila al-Faruq, Ahlam Mosteghanemi), tutte grandi divoratrici di libri, che si sono affermate (fatta eccezione di Cixous e Sebbar) fra gli ultimi anni del Novecento e i giorni nostri. Molte di queste hanno vissuto o vivono in Francia, o vi sono nate da genitori o da un genitore algerino. Un altro elemento di unità, forse non del tutto rilevante, ma non marginale è costituito dal fatto che il dossier è opera di studiose (quasi tutte piuttosto giovani), con la sola eccezione di Eloi Grasset, professore alla Harvard University. 
2 L'introduzione, ad opera di una delle curatrici Joana MAsò, docente di letteratura francese all'Università di Barcellona, evidenzia, attraverso una sintesi dei saggi raccolti nel volume, i diversi modi in cui le scrittrici algerine hanno declinato il ricorso all'intertestualità e il rapporto, sempre problematico e spesso eversivo, con l'autorità del canone. Il dossier si compone di undici articoli, in lingua francese ed inglese. Lo apre un bel saggio di Mireille RoselLo sul ruolo complesso e non privo di ambiguità assegnato da Maïssa Bey a Camus nel suo processo di formazione: si tratta di un Camus spesso reinventato dalla scrittrice per finalità proprie, la cui influenza non è pertanto subita, creando subalternità, ma autorevolmente usata per la propria liberazione e la propria crescita (Comment s'inventer un père écrivain: Albert Camus chez Maïssa Bey). A un confronto fra Maïssa Bey e Tahir Wattar è dedicato il saggio di Greta BuIss (History from Point Zero? Archival Reading in Maïss Bey's "Surtout ne te retourne pas" and Tahir Wattar's "The Earthquake"), che analizza le reazioni, profondamente diverse, dei due scrittori (attraverso i loro personaggi) di fronte al trauma provocato dal terremoto, alla perdita di punti di riferimento e di memoria e il ruolo svolto dai libri nell'opera di rimemorizzazione. Carme FIGUERoLA, dell'Università di Leida, sottolinea fin dal titolo ( $\mathrm{Du}$ livre et de la lecture dans l'oeuvre de Malika Mokeddem, pp. 23-40) l'importanza della lettura nella formazione di Malika Mokeddem e la presenza massiccia, attraverso il ricorso all'epigrafe, ma anche alla citazione, esplicita o sottintesa, dei grandi scrittori della cultura occidentale nella sua opera, un'opera che si colloca appunto nell'"entre-deux" in cui confluiscono, in maniera non "figée", ma in un costante processo di crescita, esperienze e saperi diversi: «Par ces évocations intertextuelles la romancière cherche à s'autoriser de la pensée de l'autre, en même temps qu'elle la dépasse par une déterritorialisation qui, tout compte fait, est le fruit de sa réinteprétation dans une perspective originale relevant des changements profonds opérés dans la société actuelle» (p. 39). L'articolo che segue, di Amy L. HUBBEL (Filling in the Void: Leila Sebbar's Collective Archeology of Origins, p. 41-54), è dedicato a Leila Sebbar in un'ottica memoriale che non concerne soltanto la scrittrice o la sua famiglia, ma un'esperienza più ampia, allo scopo di stabilire se l'"archeologia collettiva" di Sebbar costituisca un mezzo per riportare in vita il passato o per colmare il vuoto di un passato irrecuperabile. Denise BRAHIMI (Alham Mosteghanemi, la culture et l'histoire, pp. 55-72) si occupa in particolare di due romanzi della scrittrice Mosteghanemi, scritti in arabo tra la fine degli anni Ottanta e degli anni Novanta e tradotti in francese soltanto nel 2000: Mémoires de la chair (2002) e Les Chaos des sens (2006), entrambi ambientati nell'Algeria della post-indipendenza. Il rifiuto della colonizzazione non si disgiunge però, nella scrittrice, da una forte influenza della cultura occidentale, di cui si trova eco in entrambe le opere, senza che la presenza di due mondi in rotta di collisione generi fratture insanabili: «On imagine peut-être d'emblée que ce double aspect, histoire contemporaine de l'Algérie et culture européenne moderne en train de devenir classique, est susceptible de déboucher sur des contradictions et des déchirements. Pourtant l'évidence s'impose: il n'en est rien et les deux romans ne sont aucunement fondés sur des discordances...» (p. 56). Gemma VENTURA MUSTIENES, co-curatrice della miscellanea, sceglie di indagare le molteplici influenze letterarie che intervengono nella costruzione del secondo romanzo di Latifa Ben Mansour, La Prière de la peur (1997) (L'algérianité de Latifa Ben Mansour, un "tiers espace" identitaire, pp. 73-81). Il riferimento al "terzo spazio" di Bhabha indica la linea di lettura adottata dalla studiosa. La nozione evoca, infatti, una realtà composita, ibrida, uscita dall'esperienza coloniale, in grado di superare le contrapposizioni ideologiche tra colonizzato e colonizzatore, per dare vita a una nuova appartenenza che spezzi il 
binomio costrittivo e limitativo dell'io e dell'altro. Anche grazie all'uso sapiente del linguaggio, l'opera di Latifa Ben Mansour si pone come un'interfaccia fra mondi diversi.

Émilie CAPPELLA sceglie un classico della letteratura femminile maghrebina, un romanzo che ha suscitato negli anni molte polemiche da fronti opposti: Loin de Médine (1991) di Assia Djébar ("Loin de Médine", donner lieu de parler, pp. 83-99). La studiosa afferma che " Loin de Médine met en tension l'héritage critique du féminisme occidental avec celui des textes sacrés de l'islam, mais aussi l'héritage orientaliste avec celui de la résistance à son impérialisme» (p. 83). Il saggio si propone di evidenziare la relazione tra le voci occidentali e quelle moderniste del pensiero femminista maghrebino. In Deux cas d'écrivance ou de violence textuelle dans le "Cahier d'un retour au pays natal" d'Aimé Césaire et "Les rêveries de la femme sauvage" d'Hélène Cixous, pp. 101-118), Gwenola CARADEC mette a confronto il tema della violenza testuale in due opere molto lontane nel tempo e nello spazio e molto diverse fra loro, individuando però consonanze che sono già evidenziate dalle due citazioni degli autori, messe in esergo al suo saggio, in cui si evocano, sia pure in forme diverse, il dolore e la speranza, il disastro e la rinascita. Dal saggio emerge con evidenza il carattere performativo della violenza verbale che accomuna i due testi, entrambi fanno infatti delle parole «des "armes miraculeuses" efficaces, capables de toucher, caresser, mais aussi de réveiller autrui...» (p. 117). Il saggio che segue, di Ada BARBARO, è anch'esso focalizzato, come sottolinea il titolo, sulla violenza, non più verbale in questo caso: Memories and Violence in Fadila al-Faruq's "Ta' al-khalal": Fiction as a Tool to Construct Social Memory (pp.119-134). Il tema del romanzo (Le stigmati della violenza) indagato dalla studiosa è quello della difficile condizione femminile algerina alla fine degli anni Novanta, seguendo il percorso della giovane protagonista, che non riesce a superare il trauma della violenza subita, ma la cui scrittura autobiografica aiuta il recupero della memoria di una comunità. Il saggio che segue, di Gabriela GARCíA HUBARD, ha per oggetto l'opera filosofica di Catherine Malabou, allieva di Derrida e nata come lui in Algeria, che si è allontanata dal maestro e dal suo pensiero sulla decostruzione, per superarne il dualismo attraverso il concetto di plasticità, ricavato dai suoi studi su Hegel (Quelques réflexions: de la plasticité aux discours postcoloniaux, pp. 155-172). La Hubard analizza il pensiero di quella che è considerata un astro nascente della filosofia, in riferimento alla situazione postcoloniale: «Si le travail de Malabou nous invite à réfléchir sur la forme et l'identité (en général) à partir de la notion de plasticité, nous devrions nous demander dans quelle mesure celle-ci contribue à la formation des identités postcoloniales» (p. 157). Uno studio importante che aiuta a prendere coscienza dello sfondo filosofico di tanta narrativa algerina contemporanea, non solo femminile. Il dossier si chiude con il saggio del solo contributo maschile, quello di Eloi GRASSET, su Nina Bouraoui: ("Je ne sais plus qui je suis». L'identité indécidable et l'écriture du délire dans "Mes mauvaises pensées" de Nina Bouraoui, pp. 173-182). Il lavoro di Grasset realizza le finalità poste in premessa: «Cet article veut interroger le statut du souvenir dans l'écriture de Nina Bouraoui et, en même temps, prêter attention à la recherche de stabilisation identitaire qui se produit dans Mes mauvaises pensées (2005)» (p. 173).

Dal dossier emerge la grande vivacità della scrittura femminile algerina contemporanea e lo sforzo compiuto da tutte le scrittrici studiate per trovare un'identità che, pur essendo in discontinuità con il passato, non sia elemento di rottura ma di riconciliazione, come ben indica anche l'uso-massiccio in alcune scrittricidell'intertestualità, che è un riconoscimento dei propri debiti culturali, o la volontà di 
rompere binomi asfittici per situarsi in un "entre-deux", forse meno stabile, ma ben più vitale e fecondo. 